TRANSACTIONS OF THE

AMERICAN MATHEMATICAL SOCIETY

Volume 364, Number 2, February 2012, Pages 637-660

S 0002-9947(2011)05344-4

Article electronically published on September 14,2011

\title{
EXPONENTIAL SELF-IMPROVEMENT OF GENERALIZED POINCARÉ INEQUALITIES ASSOCIATED WITH APPROXIMATIONS OF THE IDENTITY AND SEMIGROUPS
}

\author{
ANA JIMÉNEZ-DEL-TORO
}

\begin{abstract}
The purpose of this paper is to present a general method that allows us to study exponential self-improving properties of generalized Poincaré inequalities associated with an approximation of the identity or a semigroup. In particular, we show the connection between our results and the JohnNirenberg theorem for the space $B M O$ associated with approximations of the identity and semigroups.
\end{abstract}

\section{INTRODUCTION}

It is well known that the theory of self-improving properties of Poincaré inequalities plays a fundamental role in the study of partial differential equations. The classical Sobolev embedding (see for instance [SC3]) says that $W^{1, p}\left(\mathbb{R}^{n}\right) \subset L^{p^{*}}\left(\mathbb{R}^{n}\right)$ for $1 \leq p<n$ and $p^{*}=n p /(n-p)$. That is, every function in $L^{p}\left(\mathbb{R}^{n}\right)$ with gradient in $L^{p}\left(\mathbb{R}^{n}\right)$ improves its integrability and it belongs to $L^{p^{*}}\left(\mathbb{R}^{n}\right)$. This can also be expressed via the following Poincaré-Sobolev inequality:

$$
\left(f_{Q}\left|f-f_{Q}\right|^{p^{*}} d x\right)^{1 / p^{*}} \lesssim \ell(Q)\left(f_{Q}|\nabla f|^{p} d x\right)^{1 / p} .
$$

If $p=n, W^{1, p}\left(\mathbb{R}^{n}\right) \subset \exp L^{n^{\prime}}\left(\mathbb{R}^{n}\right)$ with $n^{\prime}=n /(n-1)$ and the corresponding inequality, called Trudinger's inequality (first derived by Yudovich in [Yud]), is

$$
\left\|f-f_{Q}\right\|_{\exp L^{n^{\prime}}, Q} \lesssim\left(\int_{Q}|\nabla f|^{n} d x\right)^{1 / n} .
$$

If $n<p \leq \infty$, we have $W^{1, p}\left(\mathbb{R}^{n}\right) \subset L(1-n / p)\left(\mathbb{R}^{n}\right)$. Here $L(\alpha), \alpha \geq 0$, is the set of functions such that $f_{Q}\left|f-f_{Q}\right| \lesssim|Q|^{\alpha}$ and we also have the exponential self-improvement:

$$
\left\|f-f_{Q}\right\|_{\exp L, Q} \lesssim|Q|^{\alpha} .
$$

The case $\alpha=0$ corresponds to $B M O$ and the John and Nirenberg inequality; see JN].

Received by the editors March 13, 2009 and, in revised form, July 16, 2009.

2000 Mathematics Subject Classification. Primary 46E35; Secondary 47D06, 46E30, 42B25.

Key words and phrases. Semigroups, BMO, Poincaré inequalities, heat kernels, self-improving properties, weights, space of homogeneous type.

This research was supported by MEC Grant MTM2007-60952 and by UAM-CM Grant CCG07UAM/ESP-1664.

(C)2011 American Mathematical Society Reverts to public domain 28 years from publication 
Saloff-Coste considered further self-improvement properties of Poincaré inequalities in more general spaces such as the Riemannian manifolds; see [BCLS], SC1, [SC2, SC3]. This turns out to be useful when one studies large scale behavior of solutions of partial differential equations such as the Laplace and heat equations in these contexts. See for instance [HK1], [HK2], [Jer], [SC2], [SC3], [VSCC].

Another important contribution to this field was made by Franchi, Pérez and Wheeden in [FPW]. These authors showed that this self-improving phenomenon holds for general spaces of homogeneous type $(X, \mathrm{~d}, \mu)$ (see Section [5). Also they considered wider classes of functionals on the right-hand side and studied selfimproving properties of the following estimates:

$$
f_{B}\left|f-f_{B}\right| d x \leq a(B, f),
$$

with $a$ being a functional depending on the ball $B$, and sometimes on the function $f$. By analogy to the classical $(1,1)$-Poincaré inequality, the authors referred to (1.3) as a generalized Poincaré inequality. [FPW] introduced a general method based on the Calderón-Zygmund theory and the good- $\lambda$ inequalities discovered by Burkholder and Gundy ( $\mathrm{BG}]$ ) that allows one to establish that under mild geometric conditions on the functional $a\left(a \in D_{r}\right.$; see Section 2), inequality (1.3) encodes an intrinsic self-improvement of $L^{p, \infty}$-type for $p>1$. Moreover, it turns out that (under certain conditions) the weak-type estimate yields strong-type results. This point of view also provides the $L^{p}$-version of the John-Nirenberg inequality for functions in $B M O$.

Under a stronger condition on the functional $a$, in [MP], exponential selfimproving is obtained and this covers in particular the John-Nirenberg inequality for $B M O$ functions. They also extended some results from [HK2, Fra, [GN], $[\mathrm{Lu}, \mathrm{Bus}$. It should be pointed out that the approach used by these authors does not use any differentiability structure.

We would like to point out that in these last approaches it is key to exploit the intimate relation between the maximal operator and the sharp maximal operator. On the other hand, a new sharp maximal operator associated with an approximation of the identity $\left\{S_{t}\right\}_{t>0}$ is introduced in Ma1,

$$
M_{S}^{\#} f(x)=\sup _{B \ni x} f_{B}\left|f-S_{t_{B}} f\right| d y,
$$

where $t_{B}$ is a parameter depending on the radius of the ball $B$. This operator allows one to define the $B M O_{S}$ space, for which the John-Nirenberg theorem is also valid (see [DY]). In this way, starting with an estimate as (1.3) with $a(B, f)=C$ and where the oscillation $f-f_{B}$ is replaced by $f-S_{t_{B}} f$, a self-improving property is obtained. This new way of measuring the oscillation allows one to define new function spaces as the $B M O_{S}$ of $[\mathrm{DY}]$ and the Morrey-Campanato associated with an approximation of the identity of [DDY].

In the Euclidean setting $\mathbb{R}^{n}$ with the Lebesgue measure $d x$ (sometimes the Lebesgue measure is replaced by a Muckenhoupt weight), JM presents a method based on the Whitney covering lemma and the technique of good- $\lambda$ inequalities which allows one to obtain self-improvement in the scale of Lebesgue spaces of Poincaré-type inequalities with respect to an approximation of the identity or a 
semigroup. More precisely, let $f$ satisfy the following generalized Poincaré inequality:

$$
f_{Q}\left|f-S_{t_{Q}} f\right| d x \leq a(Q)
$$

where $t_{Q}=\ell(Q)^{m}$ and $\left\{S_{t}\right\}_{t>0}$ is an approximation of the identity or a semigroup whose kernel decays fast enough (see Section 2) and the functional $a$ satisfies a certain geometric condition $\left(a \in D_{r}\right.$; see Section 2). Then $L^{r, \infty}(Q)$ estimates are obtained for the generalized oscillation $f-S_{t_{Q}} f$. In particular, some expanded Poincare estimates that take into account the lack of localization of the approximation of the identity or the semigroup are proposed. As a consequence of this method, global pseudo-Poincaré inequalities and strong-type inequalities are obtained. Let us also mention [BJM], where this theory is extended to the so-called spaces of homogeneous type.

In this paper we continue along the lines in [JM], BJM] and the study of the exponential self-improving properties of the generalized Poincaré inequalities. That is, starting from (1.4) with a functional $a$ that is quasi-increasing (as in MP we write $a \in T_{\infty}$ ), we show an exponential-type estimate for the generalized oscillations. Also, the method yields estimates with respect to Muckenhoupt weights. We consider this problem both in the Euclidean setting and in the so-called spaces of homogeneous type. We state here our main result in the Euclidean setting and postpone the space of homogeneous type version until Part 2. The main result goes as follows (see Section 2 for the needed background and the precise definitions):

Theorem 1.1. Let $\left\{S_{t}\right\}_{t>0}$ be an approximation of the identity or a semigroup and let $a \in T_{\infty}$. Assume that $f \in \mathcal{M}$ satisfies

$$
f_{Q}\left|f-S_{t_{Q}} f\right| d x \leq a(Q)
$$

for all cubes $Q$ and where $t_{Q}=\ell(Q)^{m}$. Then, for every cube $Q$,

$$
\left\|f-S_{t_{Q}} f\right\|_{\exp L, Q} \leq C \sum_{k \geq 0} 2^{n k} g\left(2^{m(k-6)}\right) a\left(2^{k} Q\right) .
$$

Furthermore, for every $w \in A_{\infty}$ and every cube $Q$,

$$
\left\|f-S_{t_{Q}} f\right\|_{\exp L(w), Q} \leq C \sum_{k \geq 0} 2^{n k} g\left(c 2^{m k}\right) a\left(2^{k} Q\right)
$$

with $C \geq 1$ and $0<c<1$.

Remark 1.2. We would like to call attention to the fact that (1.5) is an unweighted estimate and that from it we obtain a weighted estimate for the oscillation $f-S_{t_{Q}} f$. Also, we observe that if $a$ is doubling, then the decay of $g$ easily implies that in the right-hand side of both estimates one can write $C a(Q)$.

Compare this with the approach in $\mathrm{MP}$, where a similar estimate is proved starting from (1.3). Here we use the Whitney covering lemma, in place of the Calderón-Zygmund decomposition. Since we have to take into account that the operators $S_{t}$ do not localize, this leads to some tail effects that can be found on the right-hand side of (1.6). The exponential decay assumed on the kernels gives some kind of localization, in the sense that the operators $S_{t}$ are "essentially" local, but still the values of $f$ away from a given cube matter and one needs to control 
them. Let us also point out that in our argument we need to be always localized to the given cube $Q$. Thus, when using the Whitney covering lemma and restricting to the given cube $Q$, all the involved Whitney cubes should be contained in $Q$. To do this we perform a Whitney covering lemma with respect to the dyadic grid induced by $Q$ and this is key for our purposes. Notice that in the setting of spaces of homogeneous type, we need a similar approach. Then we use Christ's dyadic sets and a Whitney covering lemma related to this dyadic structure (see Section 8.1).

The paper is organized as follows. In the first part we work in $\mathbb{R}^{n}$. The preliminaries and main definitions are in Section 2] Applications are in Section 3, and the proof of Theorem 1.1 is in Section 4. In the second part of the paper we extend the previous result to the setting of spaces of homogeneous type (see Theorem 6.1). The difference between both parts is technical: we write in detail the first part, and in the second part we omit many of the already used arguments. Preliminaries, applications and the proof of Theorem 6.1 are given respectively in Sections 5, 7 and 8

The results contained in this paper are part of the author's $\mathrm{Ph} . \mathrm{D}$. thesis, written under the supervision of Professor J.M. Martell. The author would like to thank Professor J.M. Martell for his encouragement and useful advice. The author would also like to thank the anonymous referee for suggestions to enhance the presentation of this article.

\section{Part 1. Exponential self-improvement of Poincaré inequalities in $\mathbb{R}^{n}$}

\section{Preliminaries}

Consider the Euclidean space $\mathbb{R}^{n}$ with the Lebesgue measure $d x$ (we sometimes replace the Lebesgue measure by the measure associated to a Muckenhoupt weight). We work with the infinity distance, since we manipulate cubes, that is, $|x-y|=$ $|x-y|_{\infty}=\max _{1 \leq i \leq n}\left|x_{i}-y_{i}\right|$. Obviously, all the results can be easily adapted to the Euclidean distance. We consider, without loss of generality, cubes of the form $\prod_{i=1}^{n}\left[a_{i}, a_{i}+\ell(Q)\right)$, with $a_{i} \in \mathbb{R}$ and where $\ell(Q)$ denotes the side-length of $Q$.

Given a cube $Q \subset \mathbb{R}^{n}$ and $\lambda>0$, we denote by $\lambda Q$ the cube concentric with $Q$ so that $\ell(\lambda Q)=\lambda \ell(Q)$. Also, we write

$$
f_{Q}=f_{Q} f(x) d x=\frac{1}{|Q|} \int_{Q} f(x) d x .
$$

We will use several times the following decomposition of $\mathbb{R}^{n}$ in dyadic annuli: $\mathbb{R}^{n}=$ $\bigcup_{k \geq 1} C_{k}(Q)$ with $C_{1}(Q)=2 Q$ and $C_{k}(Q)=2^{k} Q \backslash 2^{k-1} Q, k \geq 2$.

Finally, we make some conventions: $A \lesssim B$ means that the ratio $A / B$ is bounded by a constant that does not depend on the relevant variables in $A$ and $B$. Throughout this paper, the letter $C$ denotes a constant that is independent of the essential variables, but it may vary from line to line.

2.1. The space $\exp L$. This was introduced by A. Zygmund (and E. C. Titchmarsh) in 1928. See [BS] and the references therein. It appears as a natural limit of $L^{p}$ when $p \rightarrow \infty$. The space $\exp L(Q)$ is defined as the set of all measurable functions $f$ on $Q$ such that for some $C>0$,

$$
f_{Q} \exp (C|f|) d x<\infty \text {. }
$$


The localized and normalized Luxemburg norm of $\exp L(Q)$ is given by

$$
\|f\|_{\exp L, Q}=\inf \left\{\lambda>0: f_{Q}\left(\exp \frac{|f|}{\lambda}-1\right) d x \leq 1\right\} .
$$

We refer to $[\mathrm{BS}]$ and $[\mathrm{RR}]$ for more details on $\exp L$ and Orlicz spaces.

2.2. Approximations of the identity and semigroups. We work with families of linear operators $\left\{S_{t}\right\}_{t>0}$ that play the role of generalized approximations of the identity. We assume from now on that these operators commute, that is, $S_{t} \circ S_{s}=$ $S_{s} \circ S_{t}$ for every $s, t>0$. Families of operators that form a semigroup (that is, $S_{s} S_{t}=S_{s+t}$, for all $s, t>0$ ) satisfy this property. We assume that these operators admit the following integral representation:

$$
S_{t} f(x)=\int_{\mathbb{R}^{n}} s_{t}(x, y) f(y) d y,
$$

where $s_{t}(x, y)$ is a measurable function such that

$$
\left|s_{t}(x, y)\right| \leq \frac{1}{t^{n / m}} g\left(\frac{|x-y|^{m}}{t}\right)
$$

for some positive constant $m$ and for some positive, bounded and non-increasing function $g$. We assume that for all $N \geq 0$ we have

$$
\lim _{r \rightarrow \infty} r^{N} g(r)=0 .
$$

We notice that one can assume less decay on $g$ by fixing $N>0$ in the previous expression. This exponent would be taken large enough so that the estimates obtained below are not trivial (further details are left to the reader).

Let us note that the decay of $g$ yields that the integral representation of $S_{t}$ makes sense for all functions $f \in L^{p}\left(\mathbb{R}^{n}\right)$ and that the operators $S_{t}$ are uniformly bounded on $L^{p}\left(\mathbb{R}^{n}\right)$ for all $1 \leq p \leq \infty$. As in [DY], we introduce a wider class of functions for which $S_{t}$ is well defined. We define $\mathcal{M}=\bigcup_{\beta>0} \mathcal{M}_{\beta}$, where $\mathcal{M}_{\beta}$ is the set of measurable functions $f$ such that

$$
\|f\|_{\mathcal{M}_{\beta}}=\int_{\mathbb{R}^{n}}|f(x)|(1+|x|)^{-\beta} d x<\infty .
$$

In [DY], the authors establish that $\left(\mathcal{M}_{\beta},\|\cdot\|_{\mathcal{M}_{\beta}}\right)$ is a Banach space. Moreover, if $f \in \mathcal{M}$, then, for all $t, s>0, S_{t} f$ and $S_{s}\left(S_{t} f\right)$ are well defined and finite almost everywhere.

We observe that (2.1) leads to a rescaling between the parameter $t$ and the space variables. Thus, in what follows, given a cube $Q$, we write $t_{Q}=\ell(Q)^{m}$ in such a way that the parameters $t$ and $S_{t}$ are "adapted" or "scaled" to $Q$.

We present some examples of approximations of the identity and semigroups (see [JM]). We consider second order elliptic form operators: let $A$ be an elliptic $n \times n$ matrix of real and $L^{\infty}$-valued coefficients defined on $\mathbb{R}^{n}$. Associated with this matrix we define the second order divergence form operator $L f=-\operatorname{div}(A \nabla f)$, which is understood in the standard weak sense as a maximal-accretive operator on $L^{2}\left(\mathbb{R}^{n}, d x\right)$. The operator $-L$ generates a semigroup $\left\{e^{-t L}\right\}_{t>0}$ whose heat kernel has Gaussian bounds, that is, the above estimates hold with $m=2$ and $g(t)=$ $C e^{-c t}$. In dimensions $n=1,2$ one can also consider complex-valued matrices. The easiest example is given by the identity matrix in which case $L=-\Delta$ and therefore $\left\{e^{t \Delta}\right\}_{t>0}$ is the heat semigroup. We can also take the operators $S_{t}=I-\left(I-e^{-t L}\right)^{m}$ 
for some fixed $m \geq 1$, in which case we lose the semigroup property. However, we still have the commutation rule and the Gaussian decay. Thus we can apply our results to the family $\left\{S_{t}\right\}_{t>0}$. In some applications it is interesting to have $m$ large enough so that one obtains extra decay in the resulting estimates (see [JM] and the references therein).

2.3. Functionals. We consider functionals $a: \mathcal{Q} \times \mathcal{F} \longrightarrow[0,+\infty)$, where $\mathcal{Q}$ is the family of cubes in $\mathbb{R}^{n}$ and $\mathcal{F}$ is a certain family of functions. When the dependence on the functions is not of our interest, we simply write $a(Q)$.

We say that the functional $a$ is quasi-increasing or satisfies the $T_{\infty}$ condition (we write $\left.a \in T_{\infty}\right)$ if there exists a finite positive constant $C_{a}$ such that

$$
a\left(Q_{1}\right) \leq C_{a} a\left(Q_{2}\right)
$$

for every $Q_{1} \subset Q_{2}$. The infimum of the constants $C_{a}$ is denoted by $\|a\|_{T_{\infty}}$ $\left(\|a\|_{T_{\infty}} \geq 1\right)$. When $\|a\|_{T_{\infty}}=1$, we say that $a$ is increasing. This condition was introduced in MP in order to study exponential self-improving of classical Poincaré-type inequalities.

Observe that $T_{\infty}$ is stronger than $D_{r}$ (considered in [FPW] or [JM] to obtain $L^{r}$-type self-improving properties). Recall that given a Borel measure $\mu$ and $1 \leq$ $r<\infty$, we say that $a \in D_{r}(\mu)$ if there exists a constant $1 \leq C_{a}<\infty$ such that for each cube $Q$ and any family $\left\{Q_{i}\right\}_{i}$ of pairwise disjoint subcubes of $Q$, the following holds:

$$
\sum_{i} a\left(Q_{i}\right)^{r} \mu\left(Q_{i}\right) \leq C_{a}^{r} a(Q)^{r} \mu(Q) .
$$

We say that $a$ is doubling if there exists some finite positive constant $C_{a}$ such that

$$
a(2 Q) \leq C_{a} a(Q)
$$

for each cube $Q$.

2.4. Weights. A weight $w$ is a non-negative locally integrable function. For any measurable set $E$, we write $w(E)=\int_{E} w(x) d x$. Also, we set

$$
f_{Q} f d w=f_{Q} f(x) d w(x)=\frac{1}{w(Q)} \int_{Q} f(x) w(x) d x .
$$

We say that $w$ is doubling if for some finite positive constant $C_{w}$ and any cube $Q$,

$$
w(2 Q) \leq C_{w} w(Q)<\infty .
$$

A well-known collection of doubling weights is given by the Muckenhoupt classes, which are defined as follows. We say that a weight $w \in A_{p}, 1<p<\infty$, if there exists a constant $0<C<\infty$ such that for every cube $Q$,

$$
\left(f_{Q} w d x\right)\left(f_{Q} w^{1-p^{\prime}} d x\right)^{p-1} \leq C .
$$

For $p=1$, we say that $w \in A_{1}$ if there exists a constant $0<C<\infty$ such that for every cube $Q$,

$$
f_{Q} w d x \leq C w(y), \quad \text { for a.e. } y \in Q
$$


We write $A_{\infty}=\bigcup_{p \geq 1} A_{p}$. We say that $w \in R H_{p}, 1<p<\infty$, if there exists a constant $0<C<\infty$ such that for every cube $Q$,

$$
\left(f_{Q} w^{p} d x\right)^{1 / p} \leq C f_{Q} w d x
$$

We also consider the weighted exponential space $\exp L(Q, w)$ whose norm is

$$
\|f\|_{\exp L(w), Q}=\inf \left\{\lambda>0: f_{Q}\left(\exp \frac{|f|}{\lambda}-1\right) d w \leq 1\right\} .
$$

\section{Applications}

Example 1 (BMO and Morrey-Campanato spaces). As in [JM], given $\alpha \geq 0$ and $\left\{S_{t}\right\}_{t>0}$ as above (we notice that in [DY] and [DDY] these authors only consider the semigroup case), the space of Morrey-Campanato $L_{S}(\alpha)$ is defined as follows:

$$
L_{S}(\alpha)=\left\{f \in \mathcal{M}: \sup _{Q} \frac{1}{|Q|^{\alpha}} f_{Q}\left|f-S_{t_{Q}} f\right| d x<\infty\right\} .
$$

Notice that when $\alpha=0$ this space coincides with the bounded mean oscillation space $B M O_{S}$. The previous definition generalizes the classical spaces $L(\alpha)$ and $B M O$ on which $S_{t_{Q}} f$ is replaced by $f_{Q}$.

Given $f \in L_{S}(\alpha)$ with $\alpha \geq 0$, for every cube $Q$ we have that

$$
f_{Q}\left|f-S_{t_{Q}} f\right| d x \leq C|Q|^{\alpha}
$$

In this way we choose $a(Q)=|Q|^{\alpha}$ (we intentionally drop the constant as it is harmless); $a$ is clearly increasing and doubling. Then, Theorem 1.1 yields

$$
\left\|f-S_{t_{Q}} f\right\|_{\exp L, Q} \lesssim|Q|^{\alpha}
$$

for every $Q$. Also, we obtain that for every $w \in A_{\infty}$ and cube $Q$,

$$
\left\|f-S_{t_{Q}} f\right\|_{\exp L(w), Q} \lesssim|Q|^{\alpha} .
$$

On the other hand, S. Spanne Spa introduces new spaces that contain the image of $L^{p}(p>n / \alpha)$ via the fractional integral $I_{\alpha}$. These are generalizations of the classical $B M O$. We extend this definition and replace $f_{Q}$ by $S_{t_{Q}} f$. Thus, as in JM], given a non-decreasing and positive function $\varphi$ defined in $(0, \infty)$, we define $B M O_{\varphi, S}$ as follows:

$$
B M O_{\varphi, S}=\left\{f \in \mathcal{M}: \sup _{Q} \frac{1}{\varphi(\ell(Q))} f_{Q}\left|f-S_{t_{Q}} f\right| d x<\infty\right\} .
$$

Using Theorem 1.1 with $a(Q)=\varphi(\ell(Q)$ ), which is increasing (since $\varphi$ is not decreasing), we obtain that for every $f \in B M O_{\varphi, S}$ and for all cubes $Q$,

$$
\left\|f-S_{t_{Q}} f\right\|_{\exp L, Q} \leq C \sum_{k \geq 0} 2^{n k} g\left(c 2^{m k}\right) \varphi\left(2^{k} \ell(Q)\right) .
$$

In addition, if $\varphi$ is doubling (that is, $\varphi(2 t) \lesssim \varphi(t), t>0$ ), then so is $a$. This and the decay of $g$ give

$$
\left\|f-S_{t_{Q}} f\right\|_{\exp L, Q} \lesssim \varphi(\ell(Q)) .
$$

Notice that we can also get weighted versions for $A_{\infty}$ weights. 
Example 2 (Poincaré-Sobolev inequality). As in JM we consider the following expanded Poincaré-type inequality for $f \in \mathcal{M}$ :

$$
f_{Q}\left|f-S_{t_{Q}} f\right| d x \leq \sum_{k \geq 0} \alpha(k) \ell\left(2^{k} Q\right)\left(f_{2^{k} Q} h^{p} d x\right)^{1 / p}
$$

for all cubes $Q$, where $\{\alpha(k)\}_{k \geq 0}$ is a sequence of non-negative numbers and where $h$ is some non-negative measurable function; typically $h$ depends on $f$, for instance $h=C|\nabla f|$. Observe that in the classical situation, replacing $S_{t_{Q}} f$ by $f_{Q}$ and taking $h=|\nabla f|$ and $\alpha(k)=0$ for $k \geq 1$, (3.1) is nothing but a classical Poincaré-Sobolev inequality. Let us observe that if $h^{p}$ is doubling and $\alpha(k)$ decays fast enough, then (3.1) leads us to a reduced Poincaré-type inequality:

$$
f_{Q}\left|f-S_{t_{Q}} f\right| d x \leq \ell(Q)\left(f_{Q} h^{p} d x\right)^{1 / p} .
$$

However, we believe that expanded Poincaré-type inequalities are more natural in the sense that they take into account the tail effects of the approximation of the identity, in place of looking only at a somehow local term. Moreover, under certain conditions the classical Poincaré-Sobolev inequality implies (3.1) with $h=|\nabla f|$ (see $[\mathrm{JM}]$ ).

Self-improving properties of these expanded Poincaré inequalities have been studied in [JM]. In the range $1<p<n, L^{q, \infty}$ self-improving estimates are shown for every $1<q<p^{*}$. Consequently, Kolmogorov's inequality gives strong-type estimates in the same range. For $q=p^{*}$ a weak-type estimate in an Orlicz space is obtained. For the range $p \geq n$, it is pointed out that all the $D_{r}$ conditions are satisfied (since $a$ is quasi-increasing) and therefore one obtains weak and strong-type estimates in the full range $1<q<\infty$.

Here we consider the case $p \geq n$ and look for exponential estimates. As a consequence of Theorem 1.1, if $f \in \mathcal{M}$ satisfies (3.1), then

$$
\left\|f-S_{t_{Q}} f\right\|_{\exp L, Q} \lesssim \sum_{k \geq 0} \hat{\alpha}(k) \ell\left(2^{k} Q\right)\left(f_{2^{k} Q} h^{p} d x\right)^{1 / p},
$$

with $\hat{\alpha}(k)=\sum_{0 \leq l \leq k} 2^{n l} g\left(2^{m(l-6)}\right) \alpha(k-l)$ for all $k \geq 0$. Note that to use Theorem 1.1. it is enough to observe that if $p \geq n$, then

$$
a(Q)=\sum_{k \geq 0} \alpha(k) \ell\left(2^{k} Q\right)\left(f_{2^{k} Q} h^{p} d x\right)^{1 / p} \in T_{\infty} .
$$

Applying Theorem 1.1 we get the corresponding weighted version for $A_{\infty}$ weights. Note that for $p=n$, this gives a weaker version of Trudinger's inequality (1.2) with this new way of measuring the oscillation.

\section{Proof of Theorem 1.1}

4.1. Proof of 1.6. We use ideas of [MP]. We can assume that $\|a\|_{T_{\infty}}=1$. Indeed, if we take $\hat{a}(Q)=\sup _{P \subset Q} a(P)$, then $a(Q) \leq \hat{a}(Q) \leq\|a\|_{T_{\infty}} a(Q),\|\hat{a}\|_{T_{\infty}}=1$ and (1.5) holds with $a$ replaced by $\hat{a}$.

We define a new functional $\tilde{a}: \mathcal{Q} \times \mathcal{F} \longrightarrow[0,+\infty)$ given by

$$
\tilde{a}(Q)=\frac{2^{2 n}}{g(1)} \sum_{k \geq 0} 2^{n k} g\left(2^{m(k-6)}\right) a\left(2^{k} Q\right) .
$$


Observe that $a \in T_{\infty}$ implies $\tilde{a} \in T_{\infty}$ with $\|\tilde{a}\|_{T_{\infty}}=\|a\|_{T_{\infty}}=1$. Fix a cube $Q$ and assume that $\tilde{a}(Q)<\infty$. Otherwise, there is nothing to prove. We define the function

$$
G(x)=\frac{\left|f(x)-S_{t_{Q}} f(x)\right|}{\tilde{a}(Q)} \chi_{4 Q}(x) .
$$

The following result allows us to show that $G \in L^{1}\left(\mathbb{R}^{n}\right)$.

Lemma 4.1. Under the hypotheses of Theorem 1.1, for each cube $R$ and $k \geq 1$, we have

$$
f_{2^{k} R}\left|f-S_{t_{R}} f\right| d y \leq\|a\|_{T_{\infty}} a\left(2^{k} R\right) .
$$

Applying this lemma and $\tilde{a}(Q) \geq 16^{n} a(4 Q)$, we get

$$
\|G\|_{L^{1}\left(\mathbb{R}^{n}\right)}=\frac{1}{\tilde{a}(Q)} \int_{4 Q}\left|f-S_{t_{Q}} f\right| d x \leq \frac{a(4 Q)}{\tilde{a}(Q)}|4 Q| \leq \frac{1}{4^{n}}|Q|<\infty .
$$

Consider the level set

$$
\Omega=\left\{x \in \mathbb{R}^{n}: M G(x)>e\right\},
$$

where $M$ is the Hardy-Littlewood maximal function. Given that $M$ is of weak type $(1,1)$ with constant $3^{n}$, we have

$$
|\Omega| \leq \frac{3^{n}}{e}\|G\|_{L^{1}\left(\mathbb{R}^{n}\right)}<e^{-1}|Q|<\infty .
$$

Note that $\Omega$ is a level set of the lower semicontinuous function $M G$. Moreover, as we have already seen, $G \in L^{1}\left(\mathbb{R}^{n}\right)$ and $|\Omega|<\infty$. Thus, $\Omega \subsetneq \mathbb{R}^{n}$ is a proper open set.

Next we need to use the dyadic grid in $\mathbb{R}^{n}$ adapted to the cube $Q$ that consists of translations and dilations of the dyadic classical structure (see [JM]). Given $Q=\prod_{i=1}^{n}\left[a_{i}, a_{i}+\ell(Q)\right)$, the collection of dyadic cubes induced by $Q$ is denoted by $\mathcal{D}_{Q}$ and its $k$-th generation by $\mathcal{D}_{Q, k}$. That is,

$$
\mathcal{D}_{Q}=\bigcup_{k \in \mathbb{Z}} \mathcal{D}_{Q, k}
$$

where, for each $k \in \mathbb{Z}, \mathcal{D}_{Q, k}$ is the set of cubes of the form

$$
\prod_{i=1}^{n}\left[a_{i}+2^{k} m_{i} \ell(Q), a_{i}+2^{k}\left(m_{i}+1\right) \ell(Q)\right)
$$

with $m_{1}, m_{2}, \ldots, m_{n} \in \mathbb{Z}$. By construction, this dyadic decomposition has the following properties:

(i) If $R \in \mathcal{D}_{Q, k}$, then $\ell(R)=2^{k} \ell(Q)$.

(ii) If $R_{1}, R_{2} \in \mathcal{D}_{Q}$ and $R_{1} \cap R_{2} \neq \varnothing$, then either $R_{1} \subset R_{2}$ or $R_{1} \supset R_{2}$.

(iii) $\mathbb{R}^{n}=\bigcup_{R \in \mathcal{D}_{Q, k}} R$, and these cubes are pairwise disjoint.

The proofs of the following results are in [JM]. First we present a version of the Whitney covering lemma adapted to $\mathcal{D}_{Q}$. 
Theorem 4.2 ([JM] $)$. Given a cube $Q$ in $\mathbb{R}^{n}$ and $\Omega$ a proper open subset of $\mathbb{R}^{n}$, there is a family of Whitney cubes $\left\{Q_{i}\right\}_{i}$ such that

(a) $\Omega=\bigcup_{i} Q_{i}$.

(b) $\left\{Q_{i}\right\}_{i} \subset \mathcal{D}_{Q}$ and these cubes are maximal with respect to the inclusion and therefore pairwise disjoint.

(c) $\ell\left(Q_{i}\right)<\mathrm{d}\left(Q_{i}, \Omega^{c}\right) \leq 4 \ell\left(Q_{i}\right)$ and $10 Q_{i} \cap \Omega^{c} \neq \varnothing$.

When $\Omega$ is a level set of the Hardy-Littlewood maximal function, the following property holds:

Lemma 4.3 ([JM]). Fix $Q, t>0$ and $G \in L^{1}\left(\mathbb{R}^{n}\right)$. If $\Omega=\left\{x \in \mathbb{R}^{n}: M G(x)>t\right\}$ and $\left\{Q_{i}\right\}_{i}$ is its family of Whitney cubes, then, for every $k \geq 1$, we have

$$
f_{2^{k} Q_{i}} G d x \leq 10^{n} t
$$

With these results, the set $\Omega$ can be covered by the family of Whitney cubes $\left\{Q_{i}\right\}_{i} \subset \mathcal{D}_{Q}$. Furthermore, as a consequence of (4.4), for every $i$ we have

$$
\ell\left(Q_{i}\right)<\ell(Q) \text {. }
$$

For each $t>0$ and each cube $R$, we consider the set

$$
E(R, t)=\left\{x \in R:\left|f(x)-S_{t_{R}} f(x)\right|>t \tilde{a}(R)\right\} .
$$

Take $t>e$. Note that except for a set of null Lebesgue measure, we can write

$$
E(Q, t)=E(Q, t) \cap\{x \in Q: M G(x)>e\}=\bigcup_{i}\left\{x \in Q_{i} \cap Q: G(x)>t\right\} .
$$

From now on, we only consider those cubes $Q_{i}$ such that $Q_{i} \cap Q \neq \varnothing$. Therefore, since the cubes $Q_{i}$ are dyadic with respect to $Q$, (4.5) implies that $Q_{i} \subset Q$. Thus,

$$
E(Q, t)=\bigcup_{i: Q_{i} \subset Q}\left\{x \in Q_{i}:\left|f(x)-S_{t_{Q}} f(x)\right|>t \tilde{a}(Q)\right\} .
$$

Next, we replace $S_{t_{Q}} f$ by $S_{t_{Q_{i}}} f$ by using the following auxiliary result, whose proof is in Section 4.2 ,

Proposition 4.4. Under the hypotheses of Theorem 1.1, for all $x \in Q_{i}$, we have

$$
\left|S_{t_{Q_{i}}} f(x)-S_{t_{Q}} f(x)\right| \leq c_{0} \tilde{a}(Q) .
$$

Hence, if $x \in Q_{i}$, we get

$$
\left|f(x)-S_{t_{Q}} f(x)\right| \leq\left|f(x)-S_{t_{Q_{i}}} f(x)\right|+c_{0} \tilde{a}(Q) .
$$

Fix $t>C_{0}$ with $C_{0}=\max \left\{e, c_{0}\right\}$, where $c_{0}$ is the constant that appears in the previous proposition. Using that $\tilde{a}\left(Q_{i}\right) \leq \tilde{a}(Q)$ (since $a$ is increasing and $Q_{i} \subset Q$ ), we get

$$
\begin{aligned}
|E(Q, t)| & \leq \sum_{i: Q_{i} \subset Q}\left|\left\{x \in Q_{i}:\left|f(x)-S_{t_{Q_{i}}} f(x)\right|>\left(t-C_{0}\right) \tilde{a}\left(Q_{i}\right)\right\}\right| \\
& =\sum_{i: Q_{i} \subset Q}\left|E\left(Q_{i}, t-C_{0}\right)\right| .
\end{aligned}
$$


Let us define the function $\varphi:[0,+\infty) \rightarrow[0,1]$ given by

$$
\varphi(t)=\sup _{R} \frac{|E(R, t)|}{|R|} .
$$

Note that $\varphi(t) \leq 1$ for all $t \geq 0$. Furthermore, when $t>C_{0}$, (4.8) and (4.4) imply that

$$
|E(Q, t)| \leq \varphi\left(t-C_{0}\right) \sum_{i: Q_{i} \subset Q}\left|Q_{i}\right| \leq \varphi\left(t-C_{0}\right)|\Omega|<e^{-1} \varphi\left(t-C_{0}\right)|Q| .
$$

Thus, $\varphi(t)<e^{-1} \varphi\left(t-C_{0}\right)$ for all $t>C_{0}$. Therefore, iterating this estimate and using that $\varphi(t) \leq 1$ for all $t \geq 0$, we conclude that $\varphi(t) \leq e^{-\left(t / C_{0}-1\right)}$ for all $t \geq 0$. In particular, for every $t \geq 0$ we have

$$
\frac{|E(Q, t)|}{|Q|} \leq \varphi(t) \leq e^{-\left(t / C_{0}-1\right)}
$$

Hence, we pick $A>C_{0}$ and use the previous estimate to get

$$
f_{Q} \exp \left(\frac{\left|f-S_{t_{Q}} f\right|}{A \tilde{a}(Q)}\right) d x \leq \int_{0}^{\infty} e^{t} \frac{|E(Q, A t)|}{|Q|} d t \lesssim \int_{0}^{\infty} e^{-t\left(A / C_{0}-1\right)} d t<\infty .
$$

This immediately gives the desired estimate.

\subsection{Proofs of the auxiliary results.}

Proof of Lemma 4.1. Cover $2^{k} R$ with a family of pairwise disjoint subcubes $\left\{R_{i}\right\}_{i=1}^{2^{k n}}$ with side-length $\ell(R)$; thus $t_{R}=t_{R_{i}}$. As $f$ satisfies (1.5) and $a$ is increasing, we obtain

$$
\begin{aligned}
\int_{2^{k} R}\left|f-S_{t_{R}} f\right| d y & =\sum_{i=1}^{2^{k n}} \int_{R_{i}}\left|f-S_{t_{R_{i}}} f\right| d y \leq \sum_{i=1}^{2^{k n}} a\left(R_{i}\right)\left|R_{i}\right| \leq\|a\|_{T_{\infty}} a\left(2^{k} R\right) \sum_{i=1}^{2^{k n}}\left|R_{i}\right| \\
& =\|a\|_{T_{\infty}} a\left(2^{k} R\right)\left|2^{k} R\right| .
\end{aligned}
$$

Proof of Proposition 4.4. The commutation rule implies that for every $x \in Q_{i}$,

$$
\left|S_{t_{Q_{i}}} f(x)-S_{t_{Q}} f(x)\right| \leq\left|S_{t_{Q_{i}}}\left(f-S_{t_{Q}} f\right)(x)\right|+\left|S_{t_{Q}}\left(f-S_{t_{Q_{i}}} f\right)(x)\right|=I+I I,
$$

and we study each term in turn.

Pick $k_{i} \in \mathbb{Z}$ such that

$$
2^{k_{i}} \ell\left(Q_{i}\right) \leq \ell(Q)<2^{k_{i}+1} \ell\left(Q_{i}\right)
$$

Then

$$
k_{i} \geq 1 \quad \text { and } \quad 2^{k_{i}} Q_{i} \subset 2 Q .
$$

The first claim is a consequence of (4.9) together with the fact that $Q_{i} \in \mathcal{D}_{Q}$ and $\ell\left(Q_{i}\right) \leq \ell(Q) / 2$ (by (4.5)). The second claim follows from (4.9) and $Q_{i} \subset Q$.

We decompose $\mathbb{R}^{n}$ as the union of the annuli $\left\{C_{k}\left(Q_{i}\right)\right\}_{k \geq 1}$. If $x \in Q_{i}$ and $y \in C_{k}\left(Q_{i}\right)$, then

$$
\frac{|x-y|^{m}}{t_{Q_{i}}} \geq \lambda_{k}, \quad \text { where } \quad \lambda_{k}= \begin{cases}0, & \text { if } k=1, \\ 2^{m(k-3)}, & \text { if } k \geq 2 .\end{cases}
$$


In order to study $I$, we first observe that, as a consequence of (4.10), if $y \in 2^{k_{i}} Q_{i}$, then $\left|f(y)-S_{t_{Q}} f(y)\right|=G(y) \tilde{a}(Q)$ and we obtain

$$
\begin{aligned}
I & =\left|S_{t_{Q_{i}}}\left(f-S_{t_{Q}} f\right)(x)\right| \\
& \leq \frac{1}{\left|Q_{i}\right|} \int_{\mathbb{R}^{n}} g\left(\frac{|x-y|^{m}}{t_{Q_{i}}}\right)\left|f(y)-S_{t_{Q}} f(y)\right| d y \\
& \leq \frac{\tilde{a}(Q)}{\left|Q_{i}\right|} \int_{\mathbb{R}^{n}} g\left(\frac{|x-y|^{m}}{t_{Q_{i}}}\right) G(y) d y+\frac{1}{\left|Q_{i}\right|} \int_{\left(2^{k_{i}} Q_{i}\right)^{c}} \cdots d y=I_{1} \tilde{a}(Q)+I_{2} .
\end{aligned}
$$

Let us estimate $I_{1}$ : applying Lemma 4.3 .

$$
I_{1} \leq \frac{1}{\left|Q_{i}\right|} \sum_{k \geq 1} g\left(\lambda_{k}\right) \int_{C_{k}\left(Q_{i}\right)} G d y \leq \sum_{k \geq 1} 2^{n k} g\left(\lambda_{k}\right) f_{2^{k} Q_{i}} G d y \lesssim 1 .
$$

For $I_{2}$, we apply Lemma 4.1 and the fact that $C_{k}\left(Q_{i}\right) \subset 2^{k} Q_{i} \subset 2^{k-k_{i}+1} Q \subset 2^{k} Q$, for every $k \geq k_{i}$ :

$$
\begin{aligned}
I_{2} & =\frac{1}{\left|Q_{i}\right|} \sum_{k \geq k_{i}+1} \int_{C_{k}\left(Q_{i}\right)} g\left(\frac{|x-y|^{m}}{t_{Q_{i}}}\right)\left|f(y)-S_{t_{Q}} f(y)\right| d y \\
& \lesssim \sum_{k \geq k_{i}+1} 2^{n k} g\left(\lambda_{k}\right) f_{2^{k-k_{i}+1} Q}\left|f-S_{t_{Q}} f\right| d y \\
& \lesssim \sum_{k \geq k_{i}+1} 2^{n k} g\left(\lambda_{k}\right) a\left(2^{k-k_{i}+1} Q\right) \\
& \lesssim \sum_{k \geq k_{i}+1} 2^{n k} g\left(\lambda_{k}\right) a\left(2^{k} Q\right) \lesssim \tilde{a}(Q) .
\end{aligned}
$$

Plugging these into (4.11) we get $I \lesssim \tilde{a}(Q)$.

We are going to show that $I I \lesssim \tilde{a}(Q)$. Proceeding as above, by the computations in Lemma 4.1, (4.9) and (4.10), we obtain

$$
\begin{aligned}
I I & \leq \frac{1}{|Q|} \int_{\mathbb{R}^{n}} g\left(\frac{|x-y|^{m}}{t_{Q}}\right)\left|f(y)-S_{t_{Q_{i}}} f(y)\right| d y \\
& \leq \frac{g(0)}{|Q|} \int_{2^{k_{i}+2} Q_{i}}\left|f-S_{t_{Q_{i}}} f\right| d y+\frac{1}{|Q|} \sum_{k \geq k_{i}+3} g\left(\frac{\lambda_{k} t_{Q_{i}}}{t_{Q}}\right) \int_{2^{k} Q_{i}}\left|f-S_{t_{Q_{i}}} f\right| d y \\
& \lesssim a\left(2^{k_{i}+2} Q_{i}\right)+\sum_{k \geq k_{i}+3} 2^{n\left(k-k_{i}\right)} g\left(\lambda_{k} 2^{-m\left(k_{i}+1\right)}\right) a\left(2^{k} Q_{i}\right) \\
& \lesssim a\left(2^{4} Q\right)+\sum_{k \geq k_{i}+3} 2^{n\left(k-k_{i}\right)} g\left(\lambda_{k} 2^{-m\left(k_{i}+1\right)}\right) a\left(2^{k-k_{i}+1} Q\right) \\
& \lesssim a\left(2^{4} Q\right)+\sum_{k \geq 4} 2^{n k} g\left(2^{m(k-5)}\right) a\left(2^{k} Q\right) \lesssim \tilde{a}(Q)
\end{aligned}
$$

4.3. Proof of (1.7). We follow the arguments of the proof of (1.6) and so we only point out those steps where both proofs differ. First we recall that $w \in A_{\infty}$ implies that there are $1 \leq s_{0}<\infty$ and $1<s_{1} \leq \infty$ such that $w \in A_{s_{0}} \cap R H_{s_{1}}$. In particular, for any cube $Q$ and any measurable set $S \subset Q$,

$$
\frac{1}{C_{w}}\left(\frac{|S|}{|Q|}\right)^{s_{0}} \leq \frac{w(S)}{w(Q)} \leq C_{w}\left(\frac{|S|}{|Q|}\right)^{1 / s_{1}{ }^{\prime}} .
$$


The first inequality is a consequence of $w \in A_{s_{0}}$, and the second one of $w \in R H_{s_{1}}$ (see GR or Gra]).

As before, we consider $\tilde{a}$ defined in (4.1). Fix $Q$ such that $\tilde{a}(Q)<\infty$. Set $G$ to be the function given by (4.2) and $\Omega$ to be the level set of $M G$ at level $e^{s_{1}^{\prime}\left(1+\log C_{w}\right)}$. Applying Theorem 4.2 and Lemma 4.3 , we cover $\Omega$ with the family of Whitney cubes $\left\{Q_{i}\right\}_{i} \subset \mathcal{D}_{Q}$. Take the functional $\varphi:[0,+\infty) \rightarrow[0,1]$ given by

$$
\varphi(t)=\sup _{R} \frac{w(E(R, t))}{w(R)},
$$

where $E(R, t)$ is defined in (4.6). It is clear that $\varphi(t) \leq 1$ for all $t \geq 0$. Let $t>C_{0}$ with $C_{0}=\max \left\{e^{s_{1}^{\prime}\left(1+\log C_{w}\right)}, c_{0}\right\}$ and $c_{0}$ the constant in Proposition 4.4. Arguing as in (4.8), using (4.12) and $|\Omega|<e^{-s_{1}^{\prime}\left(1+\log C_{w}\right)}|Q|$, we obtain

$$
\begin{aligned}
w(E(Q, t)) & \leq \sum_{i: Q_{i} \subset Q} w\left(E\left(Q_{i}, t-C_{0}\right)\right) \leq \varphi\left(t-C_{0}\right) \sum_{i: Q_{i} \subset Q} w\left(Q_{i}\right) \\
& =\varphi\left(t-C_{0}\right) w\left(\bigcup_{i: Q_{i} \subset Q} Q_{i}\right) \\
& \leq C_{w} \varphi\left(t-C_{0}\right) w(Q)\left(\frac{\left|\bigcup_{i: Q_{i} \subset Q} Q_{i}\right|}{|Q|}\right)^{1 / s_{1}^{\prime}} \\
& <e^{-1} \varphi\left(t-C_{0}\right) w(Q) .
\end{aligned}
$$

Thus, $\varphi(t)<e^{-1} \varphi\left(t-C_{0}\right)$ for $t>C_{0}$. Iterating the previous estimate, we obtain $\varphi(t) \leq e^{-\left(t / C_{0}-1\right)}$ when $t \geq 0$. As in the proof of Theorem 1.1 the resulting estimate of the functional $\varphi$ allows us to complete the proof of (1.7).

\section{Part 2. Exponential self-improvement of Poincaré inequalities in spaces of homogeneous type}

\section{Preliminaries}

5.1. Spaces of homogeneous type. For full details and references we refer the reader to $[\mathrm{CW}$ and $\mathrm{Chr}$.

Let $(X, \mathrm{~d}, \mu)$ be a space of homogeneous type. That is, $X$ is a set equipped with a quasi-metric $d$ and a non-negative Borel measure $\mu$ satisfying the doubling condition

$$
\mu(B(x, 2 r)) \leq c_{\mu} \mu(B(x, r))<\infty
$$

for some $c_{\mu} \geq 1$, uniformly for all $x \in X$ and $r>0$. The doubling property implies that for some $c_{\mu}, n>0$ and for all $x, y \in X, r>0$ and $\lambda \geq 1$,

$$
\mu(B(x, \lambda r)) \leq c_{\mu} \lambda^{n} \mu(B(x, r))
$$

and also

$$
\mu\left(B_{2}\right) \leq c_{\mu}\left(\frac{\mathrm{r}\left(B_{2}\right)}{\mathrm{r}\left(B_{1}\right)}\right)^{n} \mu\left(B_{1}\right)
$$

for all balls $B_{1}$ and $B_{2}$ with $B_{1} \subsetneq B_{2}$.

In order to simply the computations, we assume that $X$ is unbounded and therefore $\mu(X)=\infty$. 
Let us recall that $\mathrm{d}$ being a quasi-metric on $X$ means that $\mathrm{d}$ is a function from $X \times X$ to $[0,+\infty)$ satisfying the same conditions as a metric, except for the triangle inequality that is weakened to

$$
\mathrm{d}(x, y) \leq D_{0}(\mathrm{~d}(x, z)+\mathrm{d}(z, y))
$$

for all $x, y, z \in X$ and where $1 \leq D_{0}<\infty$ is a constant independent of $x, y, z$. Unfortunately, when $D_{0}>1$, it does not follow in general that the balls are open sets. However, Macías and Segovia ( $\mathrm{MS}]$ ) proved that given any quasi-metric $\mathrm{d}$, there exists another quasi-metric $\mathrm{d}^{\prime}$ equivalent to $\mathrm{d}$ such that the metric balls defined with respect to $\mathrm{d}^{\prime}$ are open. Thus, without loss of generality, from now on we assume that the metric balls are open sets.

We use the same notation as in the first part of the paper. We replace the Lebesgue measure by the doubling and non-negative Borel measure $\mu$ and the infinity distance by the quasi-metric d. Given a ball $B \subset X$ and $\lambda>0$, we denote by $\lambda B$ the ball concentric with $B$ so that $\operatorname{r}(\lambda B)=\lambda \operatorname{r}(B)$ and by $x_{B}$ the center of $B$ and $\lambda B$.

5.1.1. Approximations of the identity and semigroups. We work with families of linear operators $\left\{S_{t}\right\}_{t>0}$ with the established properties in the first part, with the difference that now (in place of (2.1)) we assume

$$
\left|s_{t}(x, y)\right| \leq \frac{1}{\mu\left(B\left(x, t^{1 / m}\right)\right)} g\left(\frac{\mathrm{d}(x, y)^{m}}{t}\right) .
$$

As before, for any ball $B$ we write $t_{B}=\mathrm{r}(B)^{m}$. Next, as in [DY], we define $\mathcal{M}=\bigcup_{x \in X} \bigcup_{\beta>0} \mathcal{M}_{(x, \beta)}$, where $\mathcal{M}_{(x, \beta)}$ is the set of measurable functions $f$ such that

$$
\|f\|_{\mathcal{M}_{(x, \beta)}}=\int_{X} \frac{|f(y)|}{(1+\mathrm{d}(x, y))^{2 n+\beta} \mu(B(x, 1+\mathrm{d}(x, y)))} d \mu(y)<\infty .
$$

In DY], the authors establish that $\left(\mathcal{M}_{(x, \beta)},\|\cdot\|_{\mathcal{M}_{(x, \beta)}}\right)$ is a Banach space. Moreover, if $f \in \mathcal{M}$, then, for all $t, s>0, S_{t} f$ and $S_{s}\left(S_{t} f\right)$ are well defined and finite almost everywhere.

5.1.2. Functionals. We consider $a: \mathcal{B} \times \mathcal{F} \longrightarrow[0,+\infty)$, where $\mathcal{B}$ is the family of balls in $X$ and $\mathcal{F}$ is a certain family of functions. We say that $a$ is quasi-increasing or satisfies the $T_{\infty}$ condition $\left(a \in T_{\infty}\right)$ if $a\left(B_{1}\right) \leq C_{a} a\left(B_{2}\right)$ when $B_{1} \subset B_{2}$. The infimum of the positive constants $C_{a}$ is denoted by $\|a\|_{T_{\infty}}\left(\|a\|_{T_{\infty}} \geq 1\right)$. If $\|a\|_{T_{\infty}}=$ $1, a$ is said to be increasing.

5.1.3. Weights. A weight $w$ is a non-negative $\mu$-locally integrable function. For any measurable set $E$, we write $w(E)=\int_{E} w(x) d \mu(x)$. Also, we set

$$
f_{Q} f d w=f_{Q} f(x) d w(x)=\frac{1}{w(Q)} \int_{Q} f(x) w(x) d \mu(x) .
$$

The definitions and properties of weights that appear in the first part can be extended to this setting, replacing the Lebesgue measure by $\mu$ (see [ST]). 


\section{MAIN RESUlt}

As mentioned above, the goal of this part is to obtain the following extension of Theorem 1.1. In what follows, $\sigma>1$ is a sufficiently large fixed parameter (see Theorem 8.1 below).

Theorem 6.1. Let $\left\{S_{t}\right\}_{t>0}$ be as above and $a \in T_{\infty}$. Let $f \in \mathcal{M}$ be such that

$$
f_{B}\left|f-S_{t_{B}} f\right| d \mu \leq a(B)
$$

for all balls $B$. Then,

$$
\left\|f-S_{t_{B}} f\right\|_{\exp L, B} \leq C \sum_{k \geq 0} \sigma^{n k} g\left(c \sigma^{m k}\right) a\left(\sigma^{k} B\right)
$$

holds for all balls $B$. Furthermore, for all $w \in A_{\infty}$, we have

$$
\left\|f-S_{t_{B}} f\right\|_{\exp L(w), B} \leq C \sum_{k \geq 0} \sigma^{n k} g\left(c \sigma^{m k}\right) a\left(\sigma^{k} B\right) .
$$

Notice that $C \geq 1$ and $0<c<1$. If $a$ is doubling, then $a(\sigma B) \lesssim a(B)$ for all $B$, and we can write $a(B)$ on the right-hand side of the previous estimates.

\section{Applications}

In what follows, $\sigma>1$ is the sufficiently large parameter that appears in Theorem 8.1 below.

Example 3 (BMO and Morrey-Campanato spaces). As in DY] and Tan, given $\alpha \geq 0$ and $\left\{S_{t}\right\}_{t>0}$ as above (these authors only consider the semigroup case), the space of Morrey-Campanato $L_{S}(\alpha)$ is defined as follows:

$$
L_{S}(\alpha)=\left\{f \in \mathcal{M}: \sup _{B} \frac{1}{\mu(B)^{\alpha}} f_{B}\left|f-S_{t_{B}} f\right| d \mu<\infty\right\} .
$$

When $\alpha=0$ this space coincides with $B M O_{S}$.

Applying Theorem 6.1 with $a(B)=\mu(B)^{\alpha}$ (which is increasing and doubling), we have that every $f \in L_{S}(\alpha), \alpha \geq 0$, satisfies

$$
\left\|f-S_{t_{B}} f\right\|_{\exp L, B} \lesssim \mu(B)^{\alpha}
$$

for all balls $B$. Also, a weighted version of this inequality holds.

As in Example 1, one can also obtain in this setting self-improving results for $f \in B M O_{\varphi, S}(\mu)$ (where $B M O_{\varphi, S}(\mu)$ is defined as there, replacing the Lebesgue measure by $\mu$ ).

Example 4 (Poincaré-Sobolev inequality). To avoid some technicalities, we assume that all annuli are non-empty. Let $p \geq 1$. A space of homogeneous type $(X, \mathrm{~d}, \mu)$ is said to support a generalized $L^{p}$-Poincaré inequality if there exists $\mathcal{M}_{0} \subset$ $\mathcal{M}$ such that every $f \in \mathcal{M}_{0}$ satisfies

$$
f_{B}\left|f-S_{t_{B}} f\right| d \mu \leq \sum_{k \geq 0} \alpha(k) \mathrm{r}\left(\sigma^{k} B\right)\left(f_{\sigma^{k} B} h^{p} d x\right)^{1 / p}
$$

for all balls $B \subset X$, where $\{\alpha(k)\}_{k \geq 0}$ is a sequence of non-negative numbers and $h$ is some non-negative measurable function. 
Theorem 6.1 yields that if a space of homogeneous type supports a generalized $L^{p}$-Poincaré inequality with $p \geq n$ (where $n$ is the constant that appears in (5.1) and (5.2) $)$, then

$$
\left\|f-S_{t_{B}} f\right\|_{\exp L, B} \lesssim \sum_{k \geq 0} \hat{\alpha}(k) \mathrm{r}\left(\sigma^{k} B\right)\left(f_{\sigma^{k} B} h^{p} d \mu\right)^{1 / p}
$$

holds for all balls $B \subset X$, where $\hat{\alpha}(k)=\sum_{0 \leq l \leq k} \sigma^{n l} g\left(\sigma^{m(l-10)}\right) \alpha(k-l)$, for all $k \geq 0$.

To apply Theorem 6.1, it is enough to show that if $B_{1} \subset B_{2}$, then

$$
\frac{\mu\left(B_{2}\right)}{\mu\left(B_{1}\right)} \lesssim\left(\frac{\mathrm{r}\left(B_{2}\right)}{\mathrm{r}\left(B_{1}\right)}\right)^{p}
$$

as this implies that $a(B)=\sum_{k \geq 0} \alpha(k) \mathrm{r}\left(\sigma^{k} B\right)\left(f_{\sigma^{k} B} h^{p} d x\right)^{1 / p} \in T_{\infty}$. As $\mu$ is doubling and $p \geq n$, we obtain

$$
\frac{\mu\left(B_{2}\right)}{\mu\left(B_{1}\right)} \leq c_{\mu}\left(\frac{\mathrm{r}\left(B_{2}\right)}{\mathrm{r}\left(B_{1}\right)}\right)^{n} \leq c_{\mu}\left(\frac{\mathrm{r}\left(B_{2}\right)}{\mathrm{r}\left(B_{1}\right)}\right)^{p} .
$$

These inequalities appear naturally in some applications. For instance, following the notation in $\mathrm{ACDH}$, let $M$ be a non-compact Riemannian manifold with $d$ its geodesic distance and $\mu$ its volume form, which is assumed to be doubling. Let $\nabla$ and $\Delta$ be respectively the associated Riemannian gradient and Laplace-Beltrami operator. Assume that the heat kernel $p_{t}(x, y)$ of the semigroup $e^{-t \Delta}$ has Gaussian upper bounds, that is, (5.3) holds with $g(r)=C e^{-c r}$ and $m=2$. Then all our results apply in this setting. We notice that without assuming any classical Poincaré inequality, one can prove estimates such as (7.1) with $h=|\nabla f|$ or $h=|\sqrt{L}|$. For the first one, we take $S_{t}=e^{-t \Delta}$ and impose that $\sqrt{t} \nabla e^{-t \Delta}$ satisfies $L^{p}$ off-diagonal estimates. In that case one shows (7.1) with $h=|\nabla f|$ and $\alpha(k)=e^{-c 4^{k}}$. On the other hand, taking $S_{t}=I-\left(I-e^{-t \Delta}\right)^{m}$ with $m$ large enough one can obtain (7.1) with $h=|\sqrt{L} f|$ and $\alpha(k)=2^{-k \theta_{m}}$ with $\theta_{m}>0$ (by taking $m$ large, we get more decay on $\alpha(k))$. For more details we refer the reader to BJM].

\section{Proof of Theorem 6.1}

We follow the proof of Theorem 1.1. We prove (6.3) as (6.2), using the same ideas.

8.1. Step I: Dyadic case. First, we consider the following dyadic structure:

Theorem 8.1 ( $\mathrm{Chr}$ ). There exist $\sigma>4 D_{0}^{3}>1$ large enough, positive and finite constants $c_{1}, C_{1}, C_{2}$ and a countable collection $\mathcal{D}=\bigcup_{k \in \mathbb{Z}} \mathcal{D}_{k}$ of open sets $Q$ (we will refer to $Q$ as dyadic cubes and to $\mathcal{D}_{k}$ as the $k$-th generation of $\mathcal{D}$, for all $k \in \mathbb{Z}$ ) with the following properties:

(i) $\mathcal{D}_{k}$ is a countable collection of disjoint sets such that $X=\bigcup_{Q \in \mathcal{D}_{k}} Q \mu$-a.e.

(ii) Each $Q \in \mathcal{D}_{k}$ satisfies $\operatorname{diam}(Q) \leq C_{1} \sigma^{k}$, for all $k \in \mathbb{Z}$.

(iii) For each $Q \in \mathcal{D}_{k}$, there exist $x_{Q} \in Q$ and balls $B_{Q}=B\left(x_{Q}, c_{1} \sigma^{k}\right)$ and $\hat{B}_{Q}=B\left(x_{Q}, C_{1} \sigma^{k}\right)$ such that $B_{Q} \subset Q \subset \hat{B}_{Q}$, for all $k \in \mathbb{Z}$.

(iv) If $Q_{1} \in \mathcal{D}_{k_{1}}$ and $Q_{2} \in \mathcal{D}_{k_{2}}$ with $k_{1} \leq k_{2}$, then either $Q_{1} \cap Q_{2} \neq \varnothing$ or $Q_{1} \subseteq Q_{2}$. 
These sets are the analogs of the Euclidean dyadic cubes. It may help to think of $Q$ as being essentially a cube of side-length $\sigma^{k}$ and with center $x_{Q}$. The previous result (with a different notation) appears in $\mathrm{Chr}$.

From now on, we fix $\sigma>4 D_{0}^{3}$ large enough and take the dyadic structure given by Theorem 8.1 We will use the following decomposition of $X$ in dyadic annuli $X=\bigcup_{k>1} C_{k}(Q)$ with $C_{1}(Q)=\sigma \hat{B}_{Q}$ and $C_{k}(Q)=\sigma^{k} \hat{B}_{Q} \backslash \sigma^{k-1} \hat{B}_{Q}, k \geq 2$ several times. We state the following version of the Whitney covering lemma.

Theorem 8.2. Given $\Omega$ a proper open subset of $X$, there is a family of Whitney cubes $\left\{Q_{i}\right\}_{i}$ such that

(a) $\Omega=\bigcup_{i} Q_{i}$, $\mu$-almost everywhere.

(b) $\left\{Q_{i}\right\}_{i} \subset \mathcal{D}$ and these cubes are maximal with respect to the inclusion and pairwise disjoint.

(c) $0<(\sigma-1) \mathrm{r}\left(\hat{B}_{Q_{i}}\right)<\mathrm{d}\left(Q_{i}, \Omega^{c}\right) \leq \sigma^{2} \mathrm{r}\left(\hat{B}_{Q_{i}}\right)$ and $\left(C_{1} / c_{1}\right) \sigma^{3} B_{Q_{i}} \cap \Omega^{c} \neq \varnothing$.

If $\Omega$ is a level set of the maximal Hardy-Littlewood function, the following result holds.

Lemma 8.3. Given $t>0$ and $G \in L^{1}(X)$, if $\Omega=\{x \in X: M G(x)>t\}$ and $\left\{Q_{i}\right\}_{i}$ is its family of Whitney cubes, then, for every $k \geq 1$, we have

$$
f_{\sigma^{k} \hat{B}_{Q_{i}}} G d \mu \leq c_{\mu}\left(C_{1} / c_{1}\right)^{n} \sigma^{3 n} t
$$

After these preliminaries, in this part of the proof, we show that for every $1 \leq$ $\tau<\sigma^{m}$ and for every $Q \in \mathcal{D}$,

$$
\left\|f-S_{\tau t_{\hat{B}_{Q}}} f\right\|_{\exp L, Q} \lesssim \sum_{k \geq 0} \sigma^{n k} g\left(\sigma^{m(k-8)}\right) a\left(\sigma^{k} \hat{B}_{Q}\right) .
$$

As before, it is enough to assume that $\|a\|_{T_{\infty}}=1$. We present an auxiliary result; its proof is postponed until Section 8.3 .

Lemma 8.4. Under the hypotheses of Theorem 6.1, for every $1 \leq \tau<\sigma^{m}, k \geq 0$ and $R \in \mathcal{D}$, we have

$$
f_{\sigma^{k} \hat{B}_{R}}\left|f-S_{\tau t_{\hat{B}_{R}}} f\right| d \mu \leq\|a\|_{T_{\infty}} c_{\mu}^{2}\left(C_{1} / c_{1}\right)^{n} \sigma^{2 n} a\left(\sigma^{2+k} \hat{B}_{R}\right) .
$$

Consider the increasing functional $\tilde{a}: \mathcal{B} \times \mathcal{F} \longrightarrow[0,+\infty)$ given by

$$
\tilde{a}(B)=\sum_{k \geq 0} \sigma^{n k} g\left(\sigma^{m(k-6)}\right) a\left(\sigma^{k} B\right) .
$$

Fix $Q \in \mathcal{D}$ and assume that $\tilde{a}\left(\sigma^{2} \hat{B}_{Q}\right)<\infty$. Thus, the following function,

$$
G(x)=\frac{\left|f(x)-S_{\tau t_{\hat{B}_{Q}}} f(x)\right|}{\tilde{a}\left(\sigma^{2} \hat{B}_{Q}\right)} \chi_{\sigma^{2} \hat{B}_{Q}}(x),
$$

is well defined. By Lemma 8.4 and by the doubling property of the measure, we have

$$
\|G\|_{L^{1}(X)} \leq \frac{c_{\mu}^{3}\left(C_{1} / c_{1}\right)^{n} \sigma^{2 n}}{g(1)} \mu\left(\hat{B}_{Q}\right)<\infty
$$


Let $\lambda_{0}=\frac{c_{M} c_{\mu}^{4}}{g(1)}\left(\frac{C_{1}}{c_{1}}\right)^{2 n} \sigma^{4 n}>0$, where $c_{M}$ is the constant of the weak type $(1,1)$ of $M$. We consider the level set

$$
\Omega=\left\{x \in X: M G(x)>\lambda_{0}\right\}
$$

and obtain

$$
\mu(\Omega) \leq \frac{c_{M}}{\lambda_{0}}\|G\|_{L^{1}(X)} \leq \sigma^{-2 n} \mu(Q) .
$$

By Theorem 8.2 , $\Omega$ can be covered with the family of Whitney cubes $\left\{Q_{i}\right\}_{i}$ (here we use that $\Omega$ is open and $\Omega \subsetneq X$ as $\mu(X)=\infty)$. Furthermore, if $Q_{i} \cap Q \neq \varnothing$, then, for every $i$, we have $\mu\left(Q_{i}\right) \leq \sigma^{-2 n} \mu(Q), Q_{i} \subsetneq Q$,

$$
\mathrm{r}\left(\hat{B}_{Q_{i}}\right) \leq \sigma^{-2} \mathrm{r}\left(\hat{B}_{Q}\right) \quad \text { and } \quad \sigma^{2} \hat{B}_{Q_{i}} \subset \sigma \hat{B}_{Q} .
$$

The first claim follows as an immediate consequence of (8.4) and $Q_{i} \subset \Omega$. In particular, it implies that $Q_{i} \subset Q$ and $\mathrm{r}\left(\hat{B}_{Q_{i}}\right) \leq \mathrm{r}\left(\hat{B}_{Q}\right)$ (by $(i v)$ in Theorem 8.1. since $Q_{i}, Q \in \mathcal{D}$ and $Q_{i} \cap Q \neq \varnothing$ ). From this, (8.4), (5.1), (5.2) and $\sigma \geq 4 D_{0}^{3}$, we obtain

$$
\begin{aligned}
\mu\left(Q_{i}\right) & \leq \frac{c_{M} c_{\mu}^{3}\left(C_{1} / c_{1}\right)^{n} \sigma^{2 n}}{\lambda_{0} g(1)} \mu\left(\hat{B}_{Q}\right) \leq \frac{c_{M} c_{\mu}^{4}\left(C_{1} / c_{1}\right)^{n} \sigma^{2 n}}{\lambda_{0} g(1)}\left(\frac{\mathrm{r}\left(\hat{B}_{Q}\right)}{\mathrm{r}\left(B_{Q_{i}}\right)}\right)^{n} \mu\left(B_{Q_{i}}\right) \\
& \leq \frac{c_{M} c_{\mu}^{4}\left(C_{1} / c_{1}\right)^{2 n} \sigma^{2 n}}{\lambda_{0} g(1)}\left(\frac{\mathrm{r}\left(\hat{B}_{Q}\right)}{\mathrm{r}\left(\hat{B}_{Q_{i}}\right)}\right)^{n} \mu\left(Q_{i}\right) \leq \sigma^{-2 n}\left(\frac{\mathrm{r}\left(\hat{B}_{Q}\right)}{\mathrm{r}\left(\hat{B}_{Q_{i}}\right)}\right)^{n} \mu\left(Q_{i}\right),
\end{aligned}
$$

which gives $\mathrm{r}\left(\hat{B}_{Q_{i}}\right) \leq \sigma^{-2} \mathrm{r}\left(\hat{B}_{Q}\right)$. The last claim in (8.5) follows from the previous one using that $\sigma \geq 4 D_{0}^{3}$.

Next, for each $t>0$ and $R \in \mathcal{D}$, we consider the set

$$
E(R, t)=\left\{x \in R:\left|f(x)-S_{\tau t_{\hat{B}_{R}}} f(x)\right|>t \tilde{a}\left(\sigma^{2} \hat{B}_{R}\right)\right\} .
$$

Fix $t>\lambda_{0}$. Note that $\mu$-almost everywhere we have

$$
E(Q, t)=E(Q, t) \cap\left\{x \in Q: M G(x)>\lambda_{0}\right\}=\bigcup_{i}\left\{x \in Q_{i} \cap Q: G(x)>t\right\} .
$$

From now on, we only consider those cubes $Q_{i}$ such that $Q_{i} \cap Q \neq \varnothing$ and therefore $Q_{i} \subset Q$. Thus, we write

$$
E(Q, t)=\bigcup_{i: Q_{i} \subset Q}\left\{x \in Q_{i}:\left|f(x)-S_{\tau t_{\hat{B}_{Q}}} f(x)\right|>t \tilde{a}\left(\sigma^{2} \hat{B}_{Q}\right)\right\} .
$$

Next, in order to replace $S_{\tau t_{\hat{B}_{Q}}} f$ by $S_{\tau t_{\hat{B}_{Q_{i}}}} f$, we use the following auxiliary result whose proof is in Section 8.3

Proposition 8.5. Under the hypotheses of Theorem 6.1, for all $x \in Q_{i}$, we have

$$
\left|S_{\tau t_{\hat{B}_{Q_{i}}}} f(x)-S_{\tau t_{\hat{B}_{Q}}} f(x)\right| \leq c_{0} \tilde{a}\left(\sigma^{2} \hat{B}_{Q}\right) .
$$

Fix $t>C_{0}$ with $C_{0}=\max \left\{\lambda_{0}, c_{0}\right\}$. As $\tilde{a}\left(\sigma^{2} \hat{B}_{Q_{i}}\right) \leq \tilde{a}\left(\sigma^{2} \hat{B}_{Q}\right)$ (because $a$ is increasing and (8.5) holds), we obtain

$$
\begin{aligned}
\mu(E(Q, t)) & \leq \sum_{i: Q_{i} \subset Q} \mu\left(\left\{x \in Q_{i}:\left|f(x)-S_{\tau t_{\hat{B}_{Q_{i}}}} f(x)\right|>\left(t-C_{0}\right) \tilde{a}\left(\sigma^{2} \hat{B}_{Q_{i}}\right)\right\}\right) \\
& =\sum_{i: Q_{i} \subset Q} \mu\left(E\left(Q_{i}, t-C_{0}\right)\right) .
\end{aligned}
$$


As before, we define $\varphi:[0,+\infty) \rightarrow[0,1]$ given by

$$
\varphi(t)=\sup _{R \in \mathcal{D}} \frac{\mu(E(R, t))}{\mu(R)} .
$$

Note that $\varphi(t) \leq 1$ for all $t \geq 0$. This, together with (8.7), (8.4) and $\sigma>e$, implies that $\varphi(t) \leq e^{-2 n} \varphi\left(t-C_{0}\right)$ for all $t>C_{0}$. Iterating, we get $\varphi(t) \leq e^{-2 n\left(t / C_{0}-1\right)}$ for all $t \geq 0$.

To finish, we pick $A>C_{0} /(2 n)$ and using the previous estimate, we conclude that

$$
f_{Q} \exp \left(\frac{\left|f-S_{\tau t_{\hat{B}_{Q}}} f\right|}{A \tilde{a}\left(\sigma^{2} \hat{B}_{Q}\right)}\right) d \mu \leq \int_{0}^{\infty} e^{t} \frac{\mu(E(Q, A t))}{\mu(Q)} d t \leq \int_{0}^{\infty} e^{t-2 n\left(t A / C_{0}-1\right)} d t<\infty,
$$

which leads to the desired estimate.

8.2. Step $I I$ : General case. Fix a ball $B$. Let $k_{0} \in \mathbb{Z}$ be such that $C_{1} \sigma^{k_{0}} \leq$ $\mathrm{r}(B)<C_{1} \sigma^{k_{0}+1}$ and $\mathcal{I}=\left\{Q \in \mathcal{D}_{k_{0}}: Q \cap B \neq \varnothing\right\}$. Following the computations in Lemma 8.6 below, we get $\sharp \mathcal{I} \lesssim 1$ and $\mu$-almost everywhere we can write

$$
B \subset \bigcup_{Q \in \mathcal{I}} Q \subset \bigcup_{Q \in \mathcal{I}} \hat{B}_{Q} \subset \sigma B
$$

Also, each $Q \in \mathcal{I}$ satisfies $\mu\left(\hat{B}_{Q}\right) \approx \mu(B)$. Note that $\sigma^{k} \hat{B}_{Q} \subset \sigma^{k+1} B$ implies that $a\left(\sigma^{k} \hat{B}_{Q}\right) \lesssim a\left(\sigma^{k+1} B\right)$ for all $k \geq 0$. Since $t_{B}=\tau t_{Q}$ with $1 \leq \tau<\sigma^{m}$, from the preceding arguments and the Step $I$ in the proof, we obtain

$$
\begin{aligned}
\left\|f-S_{t_{B}} f\right\|_{\exp L, B} & \lesssim \sum_{Q \in \mathcal{I}}\left\|f-S_{t_{B}} f\right\|_{\exp L, Q} \\
& \lesssim \sum_{Q \in \mathcal{I}} \sum_{k \geq 0} \sigma^{n k} g\left(\sigma^{m(k-8)}\right) a\left(\sigma^{k} \hat{B}_{Q}\right) \\
& \lesssim \sum_{k \geq 0} \sigma^{n k} g\left(\sigma^{m(k-9)}\right) a\left(\sigma^{k} B\right) .
\end{aligned}
$$

\subsection{Proofs of the auxiliary results.}

Proof of Theorem 8.2. We put $\Omega=\bigcup_{k \in \mathbb{Z}} \Omega_{k}$ with

$$
\Omega_{k}=\left\{x \in \Omega: C_{1} D_{0} \sigma^{k}<\mathrm{d}\left(x, \Omega^{c}\right) \leq C_{1} D_{0} \sigma^{k+1}\right\} .
$$

Let us consider the family $\mathcal{F}$ given by

$$
\mathcal{F}=\bigcup_{k \in \mathbb{Z}}\left\{R \in \mathcal{D}_{k}: R \cap \Omega_{k+1} \neq \varnothing\right\} .
$$

We see that the cubes of $\mathcal{F}$ have the desired properties. First, let us check that they satisfy (c). If $R \in \mathcal{F}$, then, for some $k \in \mathbb{Z}, R \in \mathcal{D}_{k}$ and $R \cap \Omega_{k+1} \neq \varnothing$. Thus, there is some $x \in R \cap \Omega_{k+1}$ and

$$
C_{1} D_{0} \sigma^{k+1}<\mathrm{d}\left(x, \Omega^{c}\right) \leq D_{0}\left(\mathrm{~d}\left(R, \Omega^{c}\right)+\operatorname{diam}(R)\right) \leq D_{0}\left(\mathrm{~d}\left(R, \Omega^{c}\right)+C_{1} \sigma^{k}\right),
$$

which implies that $\mathrm{d}\left(R, \Omega^{c}\right)>C_{1} \sigma^{k}(\sigma-1)$. Besides,

$$
\mathrm{d}\left(R, \Omega^{c}\right) \leq \mathrm{d}\left(x, \Omega^{c}\right) \leq C_{1} D_{0} \sigma^{k+1} .
$$


Consequently,

$$
0<(\sigma-1) \mathrm{r}\left(\hat{B}_{R}\right)<\mathrm{d}\left(R, \Omega^{c}\right) \leq \sigma^{2} \mathrm{r}\left(\hat{B}_{R}\right) .
$$

Next, we see that $\left(C_{1} / c_{1}\right) \sigma^{3} B_{R} \cap \Omega^{c} \neq \varnothing$. The fundamental property of the infimum implies that there is $y_{0} \in \Omega^{c}$ such that

$$
\mathrm{d}\left(y_{0}, R\right)<\mathrm{d}\left(R, \Omega^{c}\right)+\mathrm{r}\left(\hat{B}_{R}\right) .
$$

Then, we use the previous inequality and (8.8) to obtain

$$
\begin{aligned}
\mathrm{d}\left(y_{0}, x_{R}\right) & \leq D_{0}\left(\mathrm{~d}\left(y_{0}, R\right)+\mathrm{r}\left(\hat{B}_{R}\right)\right)<D_{0}\left(\mathrm{~d}\left(R, \Omega^{c}\right)+2 \mathrm{r}\left(\hat{B}_{R}\right)\right) \\
& \leq D_{0}\left(\sigma^{2}+2\right) \mathrm{r}\left(\hat{B}_{R}\right) \leq\left(C_{1} / c_{1}\right) \sigma^{3} \mathrm{r}\left(B_{R}\right),
\end{aligned}
$$

which gives $y_{0} \in\left(C_{1} / c_{1}\right) \sigma^{3} B_{R} \cap \Omega^{c}$.

We now show that $\Omega=\bigcup_{R \in \mathcal{F}} R \mu$-almost everywhere. Note that $(c)$ implies that the distance of each cube $R \in \mathcal{F}$ to $\Omega^{c}$ is strictly positive; therefore, $\bigcup_{R \in \mathcal{F}} R \subset \Omega$. The other inclusion is a consequence of $\Omega=\bigcup_{k} \Omega_{k}$ together with the fact that each generation of dyadic cubes provides a $\mu$-almost everywhere partition of the space.

The problem is that the collection $\mathcal{F}$ consists of cubes that are not necessarily pairwise disjoint. In order to solve it, we select the maximal cubes of $\mathcal{F}$, eliminating those that are contained elsewhere in the collection. That is, for each cube $R \in \mathcal{F}$ select the unique maximal cube $R^{\max } \in \mathcal{F}$ which contains $R$. Such a cube exists since otherwise there exists an infinite increasing sequence of cubes $\left\{R_{k}\right\}_{k \geq 1} \subset \mathcal{F}$ containing $R$. As these cubes are in $\mathcal{F}$, they satisfy $(c)$. In particular, for every $k \geq 1$ we have

$$
\mathrm{r}\left(\hat{B}_{R_{k}}\right)<\frac{1}{\sigma-1} \mathrm{~d}\left(R_{k}, \Omega^{c}\right) \leq \frac{1}{\sigma-1} \mathrm{~d}\left(R, \Omega^{c}\right) \leq \frac{\sigma^{2}}{\sigma-1} \mathrm{r}\left(\hat{B}_{R}\right)
$$

and this leads to a contradiction. Therefore, such maximal cubes do exist and $\left\{Q_{i}\right\}_{i}$ is precisely the family of all the maximal cubes. Note that by maximality these cubes are pairwise disjoint.

Proof of Lemma 8.3. Recall that $\Omega$ is open and $\Omega \subsetneq X$ as $\mu(X)=\infty$. So $\Omega$ is a proper open set of $X$. Therefore, applying Theorem $8.2, \Omega$ can be covered with the family of Whitney cubes $\left\{Q_{i}\right\}_{i}$ and there exists $z \in\left(C_{1} / c_{1}\right) \sigma^{3} \hat{B}_{Q_{i}} \cap \Omega^{c}$. Then, if $k \geq 1$ is such that $\sigma^{k} \leq\left(C_{1} / c_{1}\right) \sigma^{3}$, using that $\mu$ is a doubling measure, we write

$$
f_{\sigma^{k} \hat{B}_{Q_{i}}} G d \mu \leq c_{\mu}\left(C_{1} / c_{1}\right)^{n} \sigma^{(3-k) n} M G(z) \leq c_{\mu}\left(C_{1} / c_{1}\right)^{n} \sigma^{2 n} t .
$$

Otherwise, when $\sigma^{k}>\left(C_{1} / c_{1}\right) \sigma^{3}$ we have

$$
f_{\sigma^{k} \hat{B}_{Q_{i}}} G d \mu \leq M G(z) \leq t .
$$

Lemma 8.6. Let $R \in \mathcal{D}_{k_{0}}$ for some $k_{0} \in \mathbb{Z}$, and set $\mathcal{J}_{k}=\left\{Q \in \mathcal{D}_{k_{0}}: Q \cap \sigma^{k} \hat{B}_{R} \neq\right.$ $\varnothing\}$ with $k \geq 0$. Then

$$
\sigma^{k} \hat{B}_{R} \subset \bigcup_{Q \in \mathcal{J}_{k}} Q \subset \bigcup_{Q \in \mathcal{J}_{k}} \hat{B}_{Q} \subset \sigma^{k+1} \hat{B}_{R}, \quad \text {-a.e. },
$$

and

$$
\# \mathcal{J}_{k} \leq c_{\mu} \sigma^{(k+2) n}\left(C_{1} / c_{1}\right)^{n}
$$


Proof. Note that [8.9) follows easily from Theorem 8.1] It is trivial to see that for every $Q \in \mathcal{J}_{k}$ we have $\sigma^{k+1} \hat{B}_{R} \subset \sigma^{k+2} \hat{B}_{Q}$. Then, all these give

$$
\begin{aligned}
& \mu\left(\sigma^{k+1} \hat{B}_{R}\right) \# \mathcal{J}_{k} \leq \sum_{Q \in \mathcal{J}_{k}} \mu\left(\sigma^{k+2} \hat{B}_{Q}\right) \leq c_{\mu} \sigma^{(k+2) n}\left(C_{1} / c_{1}\right)^{n} \sum_{Q \in \mathcal{J}_{k}} \mu(Q) \\
& \leq c_{\mu} \sigma^{(k+2) n}\left(C_{1} / c_{1}\right)^{n} \mu\left(\bigcup_{Q \in \mathcal{J}_{k}} Q\right) \leq c_{\mu} \sigma^{(k+2) n}\left(C_{1} / c_{1}\right)^{n} \mu\left(\sigma^{k+1} \hat{B}_{R}\right),
\end{aligned}
$$

and this readily implies (8.10).

Proof of Lemma 8.4. This follows as a consequence of Lemma 8.6 Fix $R \in \mathcal{D}_{k_{0}}$ for some $k_{0} \in \mathbb{Z}, k \geq 0$ and $1 \leq \tau<\sigma^{m}$. Then, by (8.9) and the fact that $a$ is increasing, we obtain

$$
\begin{aligned}
\int_{\sigma^{k} \hat{B}_{R}}\left|f-S_{\tau t_{\hat{B}_{R}}} f\right| d \mu & \leq \sum_{Q \in \mathcal{J}_{k}} \int_{\tau^{1 / m} \hat{B}_{Q}}\left|f-S_{t_{\tau^{1 / m} \hat{B}_{Q}}} f\right| d \mu \\
& \leq \sum_{Q \in \mathcal{J}_{k}} a\left(\tau^{1 / m} \hat{B}_{Q}\right) \mu\left(\tau^{1 / m} \hat{B}_{Q}\right) \\
& \leq\|a\|_{T_{\infty}} c_{\mu}\left(C_{1} / c_{1}\right)^{n} \sigma^{n} a\left(\sigma^{2+k} \hat{B}_{R}\right) \sum_{Q \in \mathcal{J}_{k}} \mu(Q) \\
& \leq\|a\|_{T_{\infty}} c_{\mu}^{2}\left(C_{1} / c_{1}\right)^{n} \sigma^{2 n} a\left(\sigma^{2+k} \hat{B}_{R}\right) \mu\left(\sigma^{k} \hat{B}_{R}\right) .
\end{aligned}
$$

Proof of Proposition 8.5. We follow the proof in the Euclidean setting, so we skip many details. The commutation rule implies that for every $x \in Q_{i}$,

$$
\begin{aligned}
\left|S_{\tau t_{\hat{B}_{Q_{i}}}} f(x)-S_{\tau t_{\hat{B}_{Q}}} f(x)\right| & \leq\left|S_{\tau t_{\hat{B}_{Q_{i}}}}\left(f-S_{\tau t_{\hat{B}_{Q}}} f\right)(x)\right|+\left|S_{\tau t_{\hat{B}_{Q}}}\left(f-S_{\tau t_{\hat{B}_{Q_{i}}}} f\right)(x)\right| \\
& =I+I I .
\end{aligned}
$$

We study each term in turn. First we pick $k_{i} \in \mathbb{Z}$ such that

$$
\sigma^{k_{i}} \mathrm{r}\left(\hat{B}_{Q_{i}}\right) \leq \mathrm{r}\left(\hat{B}_{Q}\right)<\sigma^{k_{i}+1} \mathrm{r}\left(\hat{B}_{Q_{i}}\right) .
$$

Then, by (8.5) one can see that

$$
k_{i} \geq 2 \quad \text { and } \quad \sigma^{k_{i}} \hat{B}_{Q_{i}} \subset \sigma^{2} \hat{B}_{Q} .
$$

Next, for $I$, we observe that as a consequence of (8.12), if $y \in \sigma^{k_{i}} \hat{B}_{Q_{i}}, \mid f(y)-$ $S_{\tau t_{\hat{B}_{Q}}} f(y) \mid=G(y) \tilde{a}\left(\sigma^{2} \hat{B}_{Q}\right)$ and so, arguing as in (4.11), we obtain

$$
\begin{aligned}
I \leq & \frac{\tilde{a}\left(\sigma^{2} \hat{B}_{Q}\right)}{\mu\left(B\left(x, \tau^{1 / m} \mathrm{r}\left(\hat{B}_{Q_{i}}\right)\right)\right)} \int_{X} g\left(\frac{\mathrm{d}(x, y)}{\tau^{1 / m} \mathrm{r}\left(\hat{B}_{Q_{i}}\right)}\right)^{m} G(y) d \mu(y) \\
& \quad+\frac{1}{\mu\left(B\left(x, \tau^{1 / m} \mathrm{r}\left(\hat{B}_{Q_{i}}\right)\right)\right)} \int_{\left(\sigma^{k_{i}} \hat{B}_{Q_{i}}\right)^{c}} g\left(\frac{\mathrm{d}(x, y)}{\tau^{1 / m} \mathrm{r}\left(\hat{B}_{Q_{i}}\right)}\right)^{m}\left|f(y)-S_{\tau t_{\hat{B}_{Q}}} f(y)\right| d \mu(y) \\
= & I_{1} \tilde{a}\left(\sigma^{2} \hat{B}_{Q}\right)+I_{2} .
\end{aligned}
$$

In order to estimate $I_{1}$ and $I_{2}$, we decompose $X$ as the union of dyadic annuli $\left\{C_{k}\left(Q_{i}\right)\right\}_{k \geq 1}$. Then, if $x \in Q_{i}$ and $y \in C_{k}\left(Q_{i}\right)$, we have

$$
\left(\frac{\mathrm{d}(x, y)}{\tau^{1 / m} \mathrm{r}\left(\hat{B}_{Q_{i}}\right)}\right)^{m} \geq \lambda_{k}, \quad \text { where } \quad \lambda_{k}=\left\{\begin{array}{l}
0, \text { if } k=1, \\
\sigma^{m(k-4)}, \text { if } k \geq 2 .
\end{array}\right.
$$


On the other hand, note that $\sigma^{k} \hat{B}_{Q_{i}} \subset B\left(x, \sigma^{k+1} \mathrm{r}\left(\hat{B}_{Q_{i}}\right)\right)$ and, by (5.1) and Lemma 8.3, we get

$$
I_{1} \leq \sum_{k \geq 1} g\left(\lambda_{k}\right) \frac{\mu\left(\sigma^{k} \hat{B}_{Q_{i}}\right)}{\mu\left(B\left(x, \tau^{1 / m} \mathrm{r}\left(\hat{B}_{Q_{i}}\right)\right)\right)} f_{\sigma^{k} \hat{B}_{Q_{i}}} G d \mu \lesssim \lambda_{0} \sum_{k \geq 1} g\left(\lambda_{k}\right) \sigma^{n k} \lesssim \lambda_{0} .
$$

We continue with $I_{2}$. Observe that $Q_{i} \subset Q$, and (8.11) and (8.12) imply that for every $k \geq k_{i}+1$,

$$
C_{k}\left(Q_{i}\right) \subset \sigma^{k} \hat{B}_{Q_{i}} \subset \sigma^{k-k_{i}+1} \hat{B}_{Q} \subset B\left(x, \sigma^{k+3} \mathrm{r}\left(\hat{B}_{Q_{i}}\right)\right) .
$$

Then, using the previous observations, the facts that $\mu$ is doubling and $a \in T_{\infty}$ and Lemma 8.4, we write

$$
\begin{aligned}
I_{2} & \leq \frac{1}{\mu\left(B\left(x, \tau^{1 / m} \mathrm{r}\left(\hat{B}_{Q_{i}}\right)\right)\right)} \sum_{k \geq k_{i}+1} g\left(\lambda_{k}\right) \int_{\sigma^{k-k_{i}+1} \hat{B}_{Q}}\left|f-S_{\tau t_{\hat{B}_{Q}}} f\right| d \mu \\
& \lesssim \sum_{k \geq k_{i}+1} g\left(\lambda_{k}\right) \sigma^{n k} a\left(\sigma^{k-k_{i}+3} \hat{B}_{Q}\right) \lesssim \tilde{a}\left(\sigma^{2} \hat{B}_{Q}\right) .
\end{aligned}
$$

Plugging these into (8.13), we get $I \lesssim \tilde{a}\left(\sigma^{2} \hat{B}_{Q}\right)$.

Next, we obtain the same estimate for $I I$. Notice that if $k \geq k_{i}+1$, by (8.11),

$$
\sigma^{k} \hat{B}_{Q_{i}} \subset B\left(x, \sigma^{k-k_{i}+1} \mathrm{r}\left(\hat{B}_{Q}\right)\right) \quad \text { and } \quad \sigma^{k+2} \hat{B}_{Q_{i}} \subset \sigma^{k-k_{i}+3} \hat{B}_{Q} .
$$

We use these inclusions, the fact that $\mu$ is doubling, Lemma 8.4, (8.11) and (8.12) and we have

$$
\begin{aligned}
I I \leq & \frac{g(0)}{\mu\left(B\left(x, \tau^{1 / m} \mathrm{r}\left(\hat{B}_{Q}\right)\right)\right)} \int_{\sigma^{k_{i}+1}}\left|f-S_{\tau t_{\hat{B}_{Q_{i}}}} f\right| d \mu \\
& \quad+\frac{1}{\mu\left(B\left(x, \tau^{1 / m} \mathrm{r}\left(\hat{B}_{Q}\right)\right)\right)} \sum_{k \geq k_{i}+2} g\left(\lambda_{k} \frac{t_{\hat{B}_{Q_{i}}}}{t_{\hat{B}_{Q}}}\right) \int_{\sigma^{k} \hat{B}_{Q_{i}}}\left|f-S_{\tau t_{\hat{B}_{Q_{i}}}} f\right| d \mu \\
\lesssim & a\left(\sigma^{4} \hat{B}_{Q}\right)+\sum_{k \geq k_{i}+2} g\left(\sigma^{m\left(k-k_{i}-5\right)}\right) \sigma^{n\left(k-k_{i}\right)} a\left(\sigma^{k-k_{i}+3} \hat{B}_{Q}\right) \lesssim \tilde{a}\left(\sigma^{2} \hat{B}_{Q}\right) .
\end{aligned}
$$

\section{REFERENCES}

[ACDH] P. Auscher, T. Coulhon, X.T. Duong and S. Hofmann, Riesz transforms on manifolds and heat kernel regularity, Ann. Scient. Ecole Norm. Sup. Paris 37 (2004), no. 6, 911957. MR2119242 (2005k:58043)

[BJM] N. Badr, A. Jiménez-del-Toro and J.M. Martell, $L^{p}$ self-improvement of generalized Poincaré inequalities in spaces of homogeneous type, J. Funct. Anal. 260 (2011), no. 11, 3147-3188. MR 2776565

[BCLS] D. Bakry, T. Coulhon, M. Ledoux and L. Saloff-Coste, Sobolev inequalities in disguise, Indiana J. Math. 44 (1995), 1033-1074. MR1386760 (97c:46039)

[BS] C. Bennett and R. Sharpley, Interpolation of operators, Pure and Applied Mathematics 129, Academic Press, Inc., 1988. MR928802 (89e:46001)

[BG] D.L. Burkholder and R.F. Gundy, Extrapolation and interpolation of quasi-linear operators on martingales, Acta Math. 124 (1970), 249-304. MR0440695 (55:13567)

[Bus] P. Buser, A note on the isoperimetric constant, Ann. Scient. Ecole Norm. Sup. 15 (1982), 213-230. MR683635 (84e:58076)

[Chr] M. Christ, $A T(B)$ theorem with remarks on analytic capacity and the Cauchy integral, Colloq. Math. 60/61 (1990), no. 2, 601-628. MR.1096400 (92k:42020)

[CW] R.R. Coifman and G. Weiss, Analyse harmonique non-commutative sur certains espaces homogènes, Lecture Notes in Mathematics 242, Springer-Verlag, 1971. MR0499948 $(58: 17690)$

[DDY] D. Deng, X.T. Duong and L. Yan, A characterization of the Morrey-Campanato spaces, Math. Z. 250 (2005), no. 3, 641-655. MR2179615 (2006g:42039) 
[DY] X.T. Duong and L. Yan, New function spaces of BMO type, the John-Nirenberg inequality, interpolation, and applications, Comm. Pure Appl. Math. 58 (2005), no. 10, 1375-1420. MR2162784 (2006i:26012)

[FKS] E.B. Fabes, C. Kenig and R. Serapioni, The local regularity of solution of degenerate elliptic equations, Comm. Partial Differential Equations 7 (1982), no. 1, 77-116. MR643158 (84i:35070)

[Fra] B. Franchi, Inégalités de Sobolev pour des champs de vecteurs lipschitziens, C. R. Acad. Sci. Paris, 311 (1990), no. 6, 329-332. MR1071637 (91k:46028)

[FPW] B. Franchi, C. Pérez and R.L. Wheeden, Self-improving properties of John-Nirenberg and Poincaré inequalities on spaces of homogeneous type, J. Funct. Anal. 153 (1998), no. 1, 108-146. MR1609261 (99d:42042)

[GR] J. García-Cuerva and J.L. Rubio de Francia, Weighted Norm Inequalities and Related Topics, North-Holland Math. Studies 116, North-Holland, Amsterdam, 1985. MR807149 (87d:42023)

[GN] N. Garofalo and D. Nhieu, Isoperimetric and Sobolev inequalities for CarnotCarathéodory spaces and the existence of minimal surfaces, Comm. Pure Appl. Math. 49 (1996), no. 10, 1081-1144. MR.1404326 (97i:58032)

[Gra] L. Grafakos, Classical and Modern Fourier Analysis, Pearson Education, Prentice Hall, 2004. MR2449250

[HK1] P. Hajłasz and P. Koskela, Sobolev meets Poincaré, C. R. Acad. Sci. Paris Sér. I Math. 320 (1995), no. 10, 1211-1215. MR1336257 (96f:46062)

[HK2] P. Hajłasz and P. Koskela, Sobolev met Poincaré, Mem. Amer. Math. Soc. 145 (2000), no. 688. MR $1683160(2000 \mathrm{j}: 46063)$

[HKM] J. Heinonen, T. Kilpelainen and O. Martio, Nonlinear potential theory of degenerate elliptic equations, Oxford Univ. Press, 1993. MR.1207810 (94e:31003)

[Jer] D. Jerison, The Poincaré inequality for vector fields satisfying Hörmander's condition, Duke Math. J. 53 (1986), no.2, 503-523. MR850547 (87i:35027)

[JM] A. Jiménez-del-Toro and J.M. Martell, Self-improvement of Poincaré type inequalities associated with approximations of the identity and semigroups, Preprint, 2009.

[JN] F. John and L. Nirenberg, On functions of bounded mean oscillation, Comm. Pure Appl. Math. 14 (1961), 415-426. MR0131498 (24:A1348)

[Lu] G. Lu, Embedding theorems on Campanato-Morrey spaces for vector fields of Hörmander, Approx. Theory Appl. (N.S.) (1998), no. 1, 69-80. MR.1651473|(99h:46053)

[MS] R.A. Macías and C. Segovia, Lipschitz functions on spaces of homogeneous type, Adv. in Math. 33 (1979), no.3, 257-270. MR546295 (81c:32017a)

[MP] P. MacManus and C. Pérez, Trudinger inequalities without derivatives, Trans. Amer. Math. Soc. 354 (2002), no. 5, 1997-2012. MR.1881027(2003e:46051)

[Ma1] J.M. Martell, Sharp maximal functions associated with approximations of the identity in spaces of homogeneous type and applications, Studia Math. 161 (2004), no.2, 113145. MR 2033231 (2005b:42016)

[Nir] L. Nirenberg, Estimates and uniqueness of solutions of elliptic equations, Communications on Pure and Applied Mathematics 9 (1956), 509-530. MR0091402 (19:962b)

[Tan] L. Tang, New function spaces of Morrey-Campanato type on spaces of homogeneous type, Illinois J. Math. 51 (2007), no.2, 625-644. MR2342680 (2008k:42067)

[RR] M.M. Rao and Z.D. Ren, Theory of Orlicz spaces, Monographs and Textbooks in Pure and Applied Mathematics, 146, Marcel Dekker Inc., 1991. MR1113700 (92e:46059)

[SC1] L. Saloff-Coste, Uniformly elliptic operators on Riemannian manifolds, J. Differential Geom. 36 (1992), no.2, 417-450. MR1180389 (93m:58122)

[SC2] L. Saloff-Coste, A note on Poincaré, Sobolev and Harnack inequalities, Internat. Math. Res. Notices 1992, no. 2, 27-38. MR1150597 (93d:58158)

[SC3] L. Saloff-Coste, Aspects of Sobolev-type inequalities, London Math. Soc. Lecture Notes Series 289, Cambridge University Press (2002). MR.1872526 (2003c:46048)

[Spa] S. Spanne, Some function spaces defined using the mean oscillation over cubes, Ann. Scuola. Norm. Sup. Pisa (3) 19 (1965) 593-608. MR0190729 (32:8140)

[ST] J.O. Strömberg and A. Torchinsky, Weighted Hardy spaces, Lecture Notes in Mathematics 1381, Springer-Verlag, 1989. MR:1011673 (90j:42053) 
[VSCC] N. Th. Varopoulos, L. Saloff-Coste and T. Coulhon, Analysis and geometry on groups, Cambridge Tracts in Mathematics, 100, Cambridge University Press, Cambridge, 1992. MR.1218884 (95f:43008)

[Yud] V. I. Yudovich, Dokl. Akad. Nauk SSSR 138 (1961), 805-808 (English translation: Soviet Math., no. 2-3, 1961, 746-749).

Departamento de Matemáticas, Facultad de Ciencias, Universidad Autónoma de Madrid, Campus de Cantoblanco, 28049 Madrid, Spain

E-mail address: anajimtor@hotmail.com 